\title{
BBX11 promotes red light-mediated photomorphogenic development by modulating phyB-PIF4 signaling
}

\author{
Zhaoqing Song ${ }^{1}$, Yueqin Heng ${ }^{2}$, Yeting Bian ${ }^{1}$, Yuntao Xiao' ${ }^{1}$, \\ Jiujie Liu ${ }^{1}$, Xianhai Zhao ${ }^{2,4}$, Yan Jiang ${ }^{2}$, Xing Wang Deng ${ }^{2,3 凶}$, \\ Dongqing $\mathrm{Xu}^{1}{ }_{\mathbb{B}}$
}

${ }^{1}$ State Key Laboratory of Crop Genetics and Germplasm Enhancement, National Center for Soybean Improvement, College of Agriculture, Nanjing Agricultural University, Nanjing 210095, China

${ }^{2}$ Key Laboratory of Molecular Design for Plant Cell Factory of Guangdong Higher Education Institutes, Institute of Plant and Food Sciences, Department of Biology, School of Life Sciences, Southern University of Science and Technology, Shenzhen 518055, China

${ }^{3}$ State Key Laboratory of Protein and Plant Gene Research, Peking-Tsinghua Center for Life Sciences, School of Advanced Agriculture Sciences and School of Life Sciences, Peking University, Beijing 100871, China

${ }^{4}$ Biology Department, Brookhaven National Laboratory, Upton, NY 11973, USA

Received: 30 November 2020 / Accepted: 24 February 2021 / Published online: 26 April 2021

\begin{abstract}
B (phyB) acts as the red light photoreceptor and negatively regulates the growth-promoting factor PHYTOCHROME INTERACTING 4 (PIF4) through a direct physical interaction, which in turn changes the expression of a large number of genes. phyB-PIF4 module regulates a variety of biological and developmental processes in plants. In this study, we demonstrate that B-BOX PROTEIN 11 (BBX11) physically interacts with both phyB and PIF4. BBX11 negatively regulates PIF4 accumulation as well as its biochemical activity, consequently leading to the repression of PIF4-controlled genes' expression and promotion of photomorphogenesis in the prolonged red light. This study reveals a regulatory mechanism that mediates red light signal transduction and sheds a light on phyB-PIF4 module in promoting red light-dependent photomorphognenesis.
\end{abstract}

Keywords phyB, BBX, PIF4, Photomorphogenesis, Light signaling

\section{INTRODUCTION}

Sunlight consists of a wide range of wavelength spectrum which are perceived by at least five classes of photoreceptors in plants (Galvao and Fankhauser 2015;

Zhaoqing Song and Yueqin Heng contributed equally to this work.

Supplementary Information The online version contains supplementary material available at https://doi.org/10.1007/ s42994-021-00037-2.

$\bowtie$ Correspondence: deng@pku.edu.cn (X. W. Deng), dongqingxu@njau.edu.cn (D. Xu)
Paik and Huq 2019). Photo-activated photoreceptors promptly transduce light information to downstream signaling pathway, eventually promoting various lightmediated physiological and developmental processes (Paik and Huq 2019; Jing and Lin 2020; Wei et al. 2020; Yadukrishnan et al. 2020; Yadukrishnan and Datta 2020; Yang et al. 2020). Among these, skotomorphogenesis (etiolation) and photomorphogenesis (de-etiolation) are tightly dependent on the absence or presence of light signals at the seedling stage. In darkness, germinated seeds undergo skotomorphogenetic development exhibiting elongated hypocotyls, curved apical hooks and closed cotyledons with etiolated plastids. While in 
the light, seedlings display expanded cotyledons with well-developed chloroplasts and shortened hypocotyls designated as photomorphogenesis (Jiao et al. 2007; Xu 2020). These two distinct developmental processes and their transition are precisely under the control of light signal transduction pathway involving a variety of photoreceptors, kinases, E3 ubiquitin ligase complexes and transcription factors (Paik and Huq 2019; Xu 2020; Han et al. 2020; Jing and Lin 2020; Yadav et al. 2020; Yu and Liu 2020).

phytochrome A and B (phyA and phyB) are red and far-red light photoreceptors responsible for sensing a specific wavelength region from approximately 600 to $750 \mathrm{~nm}$ (Sharrock and Quail 1989; Li et al. 2011). Of these, phyB is the predominant red light photoreceptor that initiates a plethora of red light-mediated cellular and physiological events in plants (Li et al. 2011; Paik and Huq 2019). phyB resides in the cytosol and maintains at pr form which is biologically inactive in the dark. Upon red light irradiation, pr state of phyB is converted into biologically active pfr form, subsequently shifting into the nucleus from the cytosol. pfr state of phyB interacts with a subset of PHYTOCHROME INTERACTING FACTORS (PIFs: PIF1, PIF3, PIF4 and PIF5) and triggers their rapid phosphorylation, ubiquitination and degradation within minutes (Li et al. 2011; Pham et al. 2018; Paik and Huq. 2019). Although phyB promptly promotes the degradation of PIF4 at the early stage upon red light exposure, the abundance of PIF4 maintains at a high level under the prolonged red light conditions, suggesting that PIF4 protein re-accumulates in the prolonged red light which is necessary and essential for normal seedling growth (Park et al. 2018; Yan et al. 2020). Consistently, pif4 mutant seedlings show shorter hypocotyls, whereas transgenic seedlings over-expressing PIF4 exhibit longer hypocotyls than wide-type (WT) in the constant red light (Huq and Quail. 2002). As a b-HLH type transcription factor, PIF4 directly binds to the promoter regions of multiple regulators of auxin biosynthesis and signaling to promote cell elongation and hypocotyl growth in plants (Franklin et al. 2011; Sun et al. 2012, 2013). Thus PIF4 represents a key node in phyB signaling through which plants integrate diverse internal and external cues to modulate seedling development.

B-box proteins (BBXs), which are characterized by the presence of one or two conserved B-box domains at the $\mathrm{N}$-terminal region, play critical roles in light signaling, photoperiodic flowering, pigment accumulation and distinct hormonal signaling pathways (Gangappa and Botto. 2014; Vaishak et al. 2019; Song et al. 2020a; Xu. 2020). There are 32 BBXs members in Arabidopsis which are classified into five subfamilies according to their respective domain structures and features (Khanna et al. 2009). Increasing studies have revealed that a group of BBXs regulate photomorphogenic development. BBX4 and BBX21-BBX23 are positive regulators of light signaling, whereas BBX19, BBX24, BBX25 and BBX28-BBX32 inhibit photomorphogenesis (Datta et al. 2006, 2007, 2008; Holtan et al. 2011; Fan et al. 2012; Gangappa et al. 2013; Wang et al. 2015; Xu et al. 2016, 2018; Zhang et al. 2017a, b; Job et al. 2018; Lin et al. 2018; Heng et al. 2019a,b; Yadav et al. 2019; Bursch et al. 2020; Song et al. 2020b; Wu et al. 2020). BBX4 specifically promotes phyB-mediated signaling (Datta et al. 2006; Heng et al. 2019a). Red light-activated phyB interacts with and stabilizes BBX4 that forms inactive heterodimers with PIF3 to repress its biochemical action and PIF3-controlled gene expression, thereby promoting photomorphogenesis in the red light (Heng et al. 2019a). BBX19 facilitates the CONSTITUTIVELY PHOTOMORPHOGENIC 1 (COP1)-mediated degradation of EARLY FLOWERING 3 (ELF3) which is a core member of the evening complex, consequently leading to the increase of PIF4 and PIF5 as well as hypocotyl growth (Wang et al. 2015). Unlike BBX4 and BBX19, the other BBXs form a transcriptional regulatory network with ELOGATED HYPOCOTYL 5 (HY5) by affecting its transcription and/or biochemical activity to negatively or positively regulate photomorphogenic development (Datta et al. 2007, 2008; Holtan et al. 2011; Fan et al. 2012; Gangappa et al. 2013; Xu et al. 2016, 2018; Zhang et al. 2017b; Lin et al. 2018; Heng et al. 2019b; Yadav et al. 2019; Bursch et al. 2020; Song et al. 2020b). As such, different BBXs are considered to exert distinct modes of action in the control of photomorphogenesis (Vaishak et al. 2019; Xu 2020; Song et al. 2020a).

Recently, we have revealed that BBX11, BBX21 and HY5 form a feedback loop that promotes photomorphogenesis. Loss of BBX11 function mutants are specifically hyposensitive to the monochromatic red light, but not to the blue and far-red light (Zhao et al. 2020), suggesting that BBX11 is a key component of phyB signaling. Here, we demonstrate that BBX11 physically interacts with both phyB and PIF4. BBX11 not only promotes the degradation of PIF4 but also inhibits its biochemical activity in the red light. Consequently, BBX11 modulates the phyB-PIF4-mediated signaling to promote photomorphogenic development in plants. 


\section{RESULTS}

\section{BBX11 interacts with red light photoreceptor phyB}

Our previous studies suggest that BBX11 is a positive regulator of phyB signaling (Zhao et al. 2020). To explore the mechanism and molecular context of BBX11 action in this process, we first performed yeast-two hybrid experiments to test whether BBX11 directly interacts with red light photoreceptor phyB. As BINDING DOMAIN (BD)-fused phyB exhibited self-activation activity in yeast cells (Supplemental Fig. 1), we thus constructed BD-phyB-N (1-652 aa) containing a chromophore binding domain, BD-phyB-C containing a PRD and a HKRD domain (652-1172 aa) for this assay (Fig. 1A). ACTIVATING DOMAIN (AD)-fused BBX11 could interact with BD-phyB-C (652-1172 aa) but not BD-phyB-N (1-651 aa) (Fig. 1B). The phyB C-terminus contains one PRD and one HKRD domain. To examine the exact domain in phyB responsible for the association with BBX11, BD-phyB-C1 containing a HKRD (910-1172 aa) and BD-phyB-C2 containing a PRD (652-910 aa) were generated (Fig. 1A; Supplemental Fig. 1A). BD-
phyB-C2 (652-910 aa) showed self-activation activity (Supplemental Fig. 1B), and BD-phyB-C1 (910-1172 aa) did not interact with AD-BBX11 in yeast cells (Fig. 1B). These results suggest that the $C$-terminal region of phyB is required for the interaction with BBX11. To verify the phyB-BBX11 interaction, we employed a co-immunoprecipitation (Co-IP) assay and transiently co-expressed 35S:myc-BBX11 alone or together with 35S:phyB-Flag in the Nicotiana benthamian leaves. myc-BBX11 co-immunoprecipitated phyB-Flag as detected by immunoblot analysis (Fig. 1C). Next, we performed firefly luciferase complementation imaging (LCI) assays and fused spilt $\mathrm{N}$-terminal LUC (nLUC) and C-terminal LUC (cLUC) with phyB (phyB-nLUC) and BBX11 (cLUC-BBX11) respectively. The phyB-nLUC and cLUC-BBX11 were transiently co-expressed in Nicotiana benthamiana leaves. The Nicotiana benthamiana plants were kept in darkness for $1 \mathrm{~d}$, and then transferred to darkness or red light for additional $2 \mathrm{~d}$. We detected the strong LUC influence signals in Nicotiana benthamiana leaves with red light irradiation, but not in those leaves kept in darkness (Fig. 1D). The LUC signals were not detectable when transiently co-expressed negative controls nLUC and cLUC, phyB-nLUC and cLUC, or nLUC
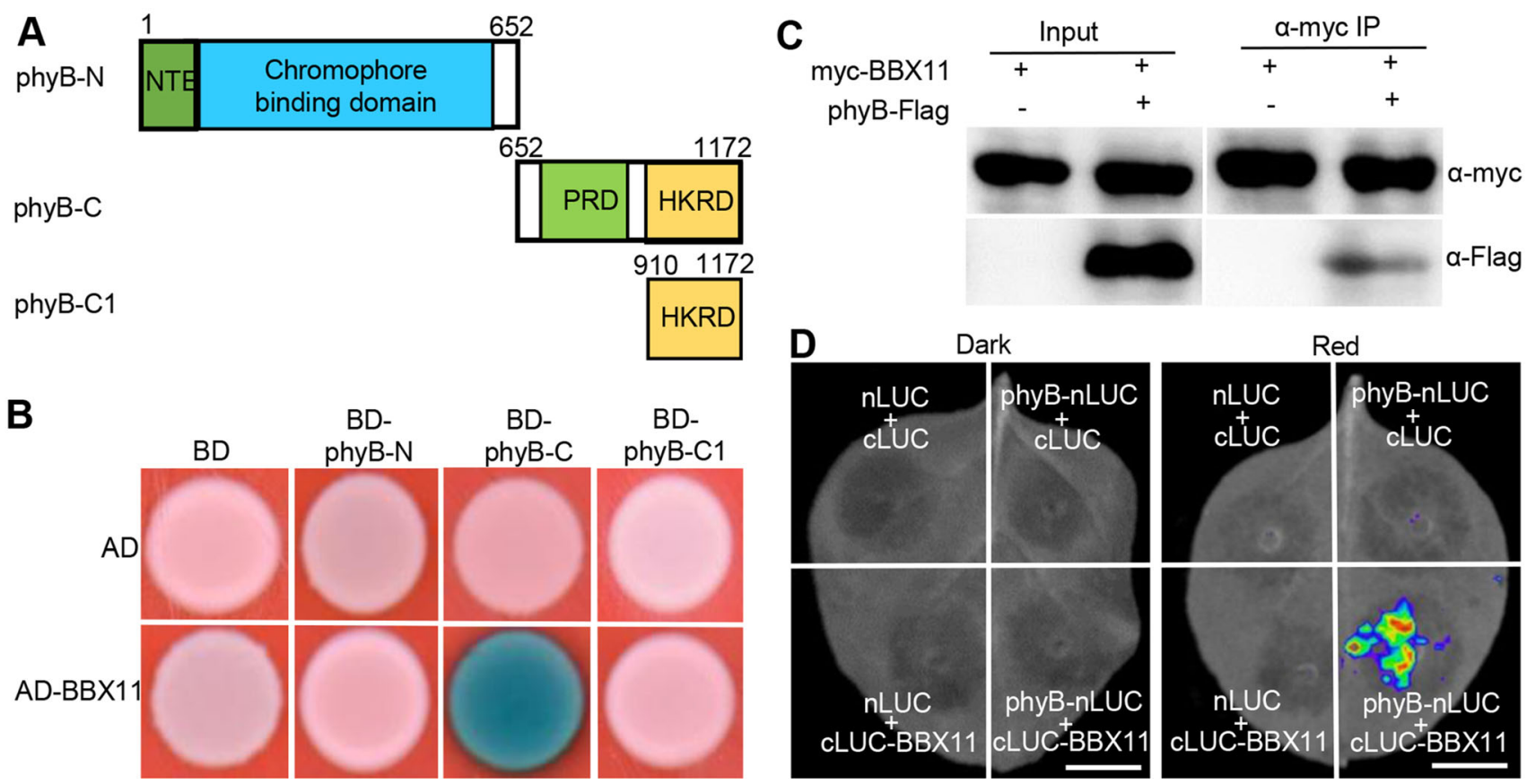

Fig. 1 phyB interacts with BBX11 both in yeast and living plant cells. A Schematic diagram of various constructs used in the yeast twohybrid assays. Numbers indicate the amino acid positions in phyB. B Yeast two-hybrid interactions between the various truncated phyB and BBX11 proteins. C Co-immunoprecipitation analysis showing that myc-BBX11 interacts with phyB-Flag. Total protein was extracted from wild tobacco leaves transiently expressing 35S:myc-BBX11 alone or together with 35S:phyB-Flag. The immunoprecipitates were detected using anti-myc and anti-Flag antibodies. D LCI assays showing the interaction of phyB with BBX11. Full-length phyB and BBX11 were fused to the split N- or C- terminal (nLUC or cLUC) fragments of LUC. The phyB-nLUC and cLUC-BBX11 were transiently coexpressed in Nicotiana benthamiana leaves and incubated in darkness for 1 day. Then the Nicotiana benthamiana plants were shifted in darkness or red light $\left(94 \mu \mathrm{mol} / \mathrm{m}^{2} / \mathrm{s}\right)$ respectively for an additional $2 \mathrm{~d}$. nLUC and cLUC were used as negative controls. Bar $=1 \mathrm{~cm}$ 
and CLUC-BBX11 in the Nicotiana benthamian leaves (Fig. 1D). Together, these results suggest that red light photoreceptor phyB associates with BBX11 in yeast and living plant cells, and the association of phyB with BBX11 might be dependent on the presence of red light.

\section{BBX11 physically interacts with PIF4}

Photo-activated phyB directly interacts with downstream signaling components PIFs in mediating a variety of light-dependent development in plants (Leivar and Monte 2014), we thus examined whether BBX11 associates with PIFs. BBX11, which is a transcription factor, exhibited self-transactivation activity in yeast cells and its middle portion is likely responsible for this action (Supplemental Fig. 2), which is consistent with previous studies that the middle portion of BBXs possesses transcriptional activation activity (Zhang et al. 2014; Heng et al. 2019a; Bursch et al. 2020). We thus generated a BD-BBX11 $\Delta(98-212$ aa) construct lacing transactivation activity (Supplemental Fig. 2). Yeast-two hybrid assays showed that BBX11 $\Delta(98-212$ aa) specifically interacted with PIF4, but not with PIF1, PIF3 and PIF5 (Fig. 2A). LCI experiments showed that co-expression of PIF4-nLUC and cLUC-BBX11 in Nicotiana benthamiana leaves clearly produced LUC signals (Fig. 2B).When we transiently co-expressed negative controls nLUC and cLUC, PIF4-nLUC and cLUC, or nLUC and CLUC-BBX11 in Nicotiana benthamiana leaves, the LUC signals were not observed in the same detection system (Fig. 2B). Co-IP assays were employed to further verify these results. 35S:myc-BBX11 alone or together with 35S:PIF4-Flag were transiently expressed in the Nicotiana benthamian leaves. As shown in Fig. 2C, mycBBX11 could pull down PIF4-Flag. Taken together, these results suggest that BBX11 physically interacts with PIF4 in planta.

\section{BBX11 promotes the degradation of PIF4 in the prolonged red light}

To assess whether phyB regulates the abundance of BBX11, we introduced the phyb-9 mutation into YFPBBX11 \#8 by genetic crossing. YFP-BBX11 \#8 and YFP$B B X 11$ \#8 phyb-9 grown in the constant red light accumulated similar YFP-BBX11 abundance (Supplemental Fig. 3A). In addition, comparable YFP-BBX11 protein levels were detected in the dark-grown YFP-BBX11 \#8 and YFP-BBX11 \#8 phyb-9 upon transferred to red light irradiation for various time periods $(0,15,30$ and
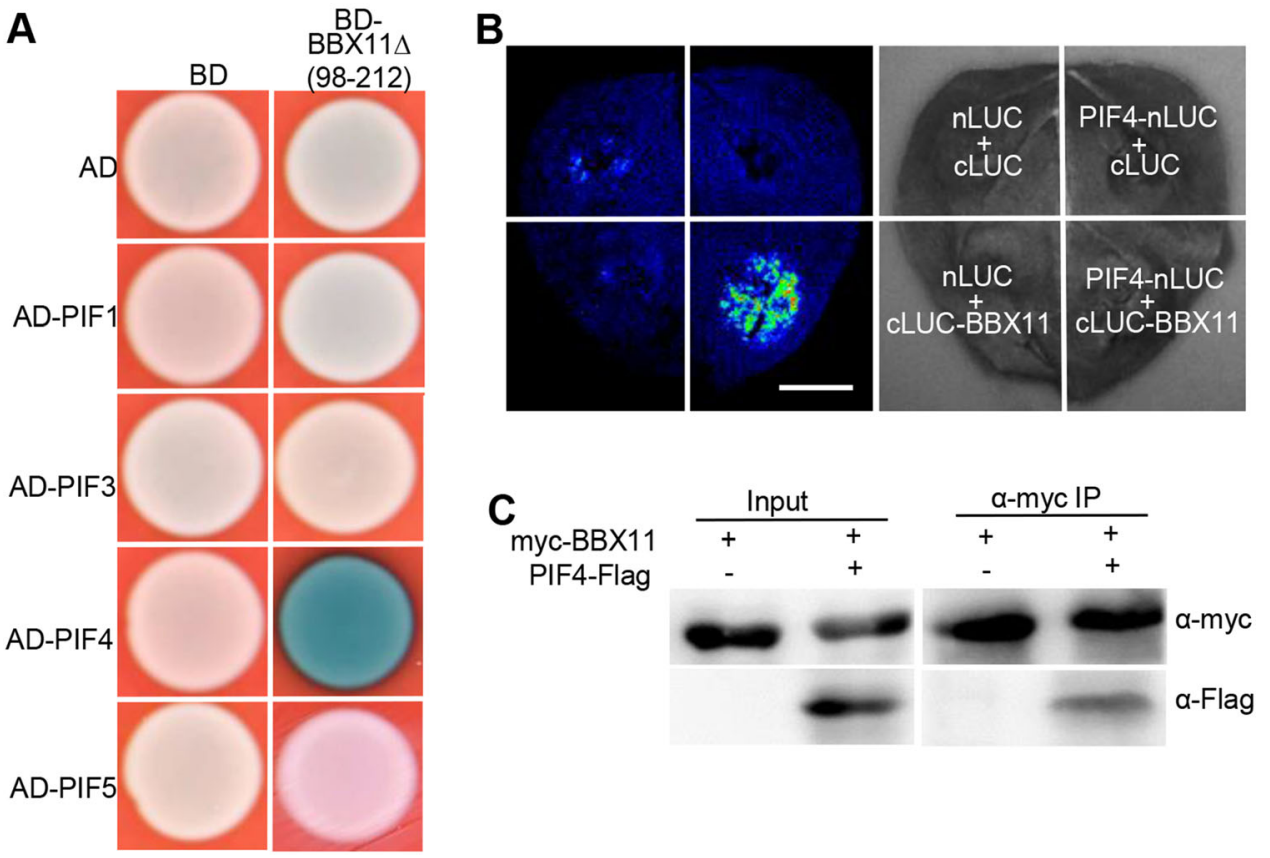

Fig. 2 BBX11 physically interacts with PIF4. A Yeast two-hybrid assays showing that BBX11 $\Delta$ (98-212) lacking transactivation domain interacts with PIF4, but not PIF1, PIF3 and PIF5. B LCI assays showing the interaction of PIF4 with BBX11. Full-length PIF4 and BBX11 were fused to the split N- or C- terminal (nLUC or cLUC) fragments of LUC. nLUC and cLUC were used as negative controls. Bar $=1 \mathrm{~cm}$. C Co-immunoprecipitation analysis showing that myc-BBX11 interacts with PIF4-Flag. Total protein was extracted from wild tobacco leaves transiently expressing 35S:myc-BBX11 alone or together with 35S:PIF4-Flag. The immunoprecipitates were detected using antimyc and anti-Flag antibodies 
60 min) (Supplemental Fig. 3B). These results indicate that phyB may not affect the accumulation of BBX11 in the red light.

Red light activated-phyB promotes the rapid degradation of PIF4 (Huq and Quail 2002; Lorrain et al. 2008; Zhang et al. 2017a), we, therefore, examined the PIF4 protein levels in dark-grown $b b \times 11$ single mutants and $B B X 11$ overexpressors upon transferred to red light for various time points $(0,0.5$ and $1 \mathrm{~h})$. Etiolated Col-0, bbx11-1 and YFP-BBX11 \#8 seedlings accumulated comparable PIF4 proteins. Upon transferred to red light for 0.5 or $1 \mathrm{~h}$, PIF4 proteins were barely detectable in all these seedlings (Fig. 3A), suggesting that BBX11 likely has a litter effect on the phyB- and red lighttriggered rapid degradation of PIF4 (Fig. 3A). Following prolonged red light irradiation, PIF4 re-accumulates and
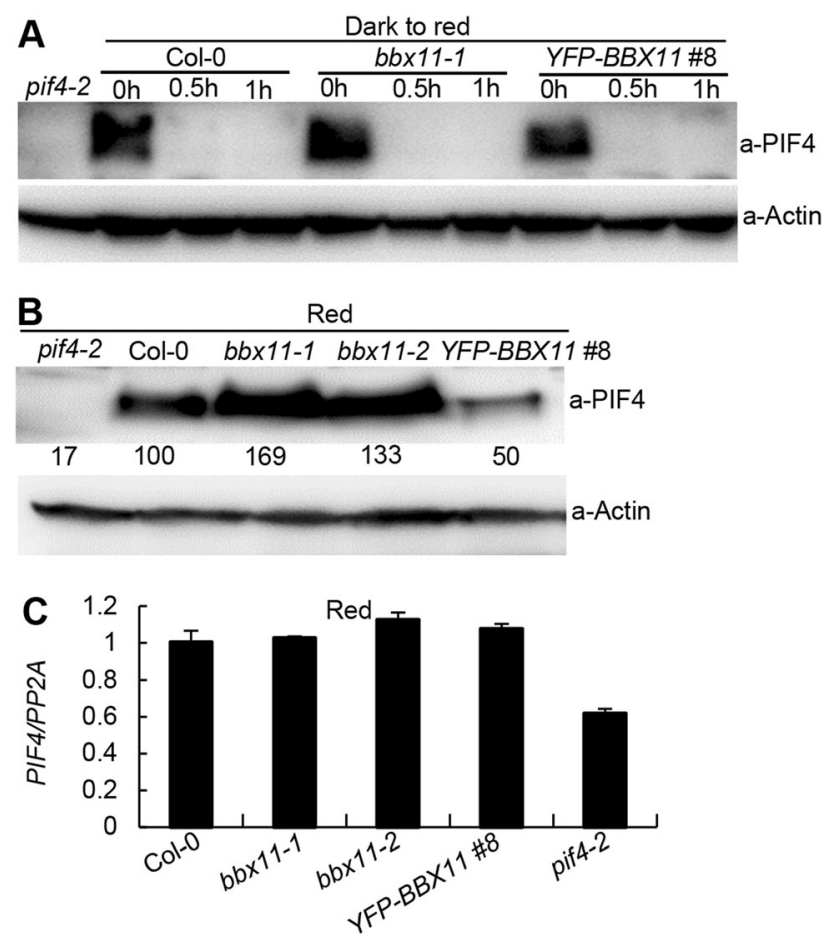

Fig. 3 BBX11 represses the reaccumulation of PIF4 in the prolonged red light. A Immunoblots showing the PIF4 protein levels in Col-0, $b b \times 11-1$ and YFP-BBX11 \#8 seedlings grown in darkness for 4 days, then transferred to red $\left(94 \mu \mathrm{mol} / \mathrm{m}^{2} / \mathrm{s}\right)$ light as indicated time points $(0,0.5$, and $1 \mathrm{~h})$. pif4-2 served as a negative control. Anti-Actin was used as a loading control. B Immunoblots showing the PIF4 protein levels in Col-0, bbx111, bbx11-2, YFP-BBX11 \#8 seedlings grown in the continuous red ( $94 \mu \mathrm{mol} / \mathrm{m}^{2} / \mathrm{s}$ ) light for 4 days. pif4-2 served as a negative control. Anti-Actin was used as a loading control. Numbers below the immunoblots indicate the relative intensities of PIF4 bands normalized to those of Actin, and the ratio was set to 100 for that in Col-0. C The transcription levels of PIF4 in Col-0, bbx11-1, $b b \times 11-2, Y F P-B B X 11$ \#8 seedlings grown in the continuous red $\left(94 \mu \mathrm{mol} / \mathrm{m}^{2} / \mathrm{s}\right)$ light for 4 days, as determined by real-time qRTPCR. pif4-2 served as a negative control maintains at a high level to promote seedling growth (Zhang et al. 2017a; Yan et al. 2020). We next investigated the PIF4 protein levels in Col-0, bbx11-1, bbx11-2 and YFP-BBX11 \#8 seedlings grown in constant red light for $4 \mathrm{~d}$. Two independent $b b \times 11$ (bbx11-1 and $b b \times 11-2$ ) mutant seedlings accumulated obviously more PIF4 abundance, whereas the PIF4 protein levels in YFP$B B X 11 \# 8$ were clearly less abundant compared to those in Col-0, $b b \times 11-1$ and $b b \times 11-2$ (Fig. 3B), suggesting that BBX11 promotes the degradation of PIF4 in the prolonged red light.

As BBX11 acts as a transcription factor (Zhao et al. 2020), we thus examined whether BBX11 regulates the PIF4 at the transcriptional level. The transcript levels of PIF4 were not significantly altered in $b b \times 11-1, b b \times 11-2$ and YFP-BBX11 \#8 compared to that in Col-0 grown in the constant red light, while the expression of PIF4 was drastically decreased in the pif4-2 mutant seedlings (Fig. 3C), suggesting that BBX11 may not affect the expression of PIF4 in red light-grown seedlings.

\section{BBX11 enhances the interaction between phyB and PIF4}

The interaction of phyB with PIF4 is required prior to the degradation of PIF4 upon red light irradiation (Lorrain et al. 2008). Considering that BBX11 not only interacts with both phyB and PIF4 but also promotes the degradation of PIF4 (Figs. 1, 2 and 3A), we carried out yeast-three hybrid assays to examine whether BBX11 affects the interaction of phyB with PIF4. phyB interacted with the PIF4 in yeast cells as revealed by relative $\beta$-Galactosidase activity (Fig. $4 \mathrm{~A}$ ), which is consistent with a previous study (Huq and Quail 2002). The value of relative $\beta$-Galactosidase activity was significantly increased in the presence of BBX11 (Fig. 4A). Next, we employed LCI assays to verify these results. The LUC signals were clearly detectable when transiently co-expressed phyB-nLUC and cLUC-PIF4 in the Nicotiana benthamiana leaves. While co-expression of phyB-nLUC and cLUC-PIF4 together with BBX11 in the same leaves led to a markedly increase in the LUC signals as detected in the same experimental system (Fig. 4B). Together, these results suggest that BBX11 enhances the interaction of phyB with PIF4 in both yeast and living plant cells.

\section{BBX11 represses PIF4 biochemical activity}

Given that BBX11 physically interacts with PIF4, we wonder whether BBX11 has any effect on the PIF4 activity. The Nicotiana benthamiana leaves when transiently expressed PIF4 alone or together with BBX11 

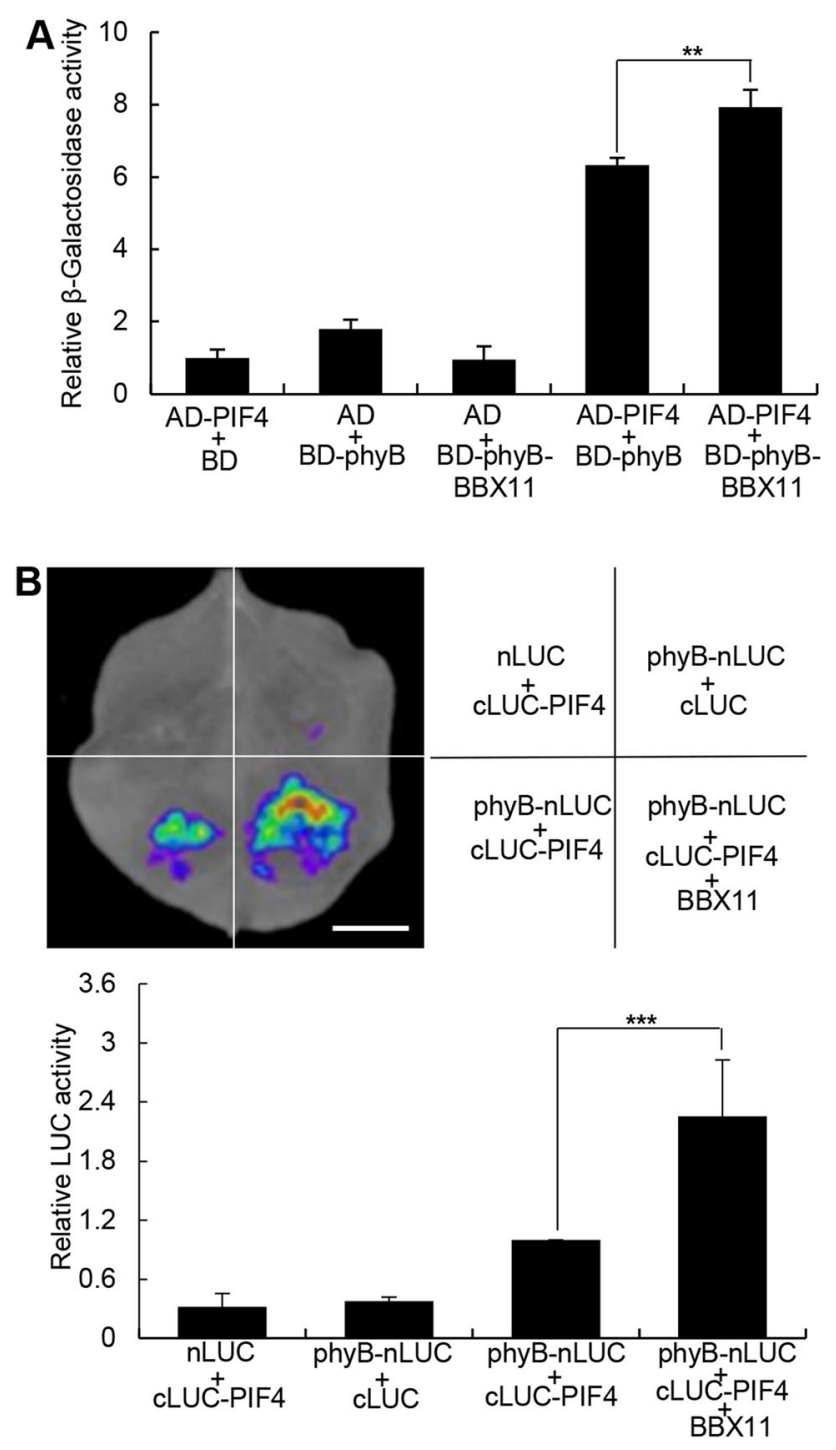

Fig. 4 BBX11 enhances the interaction of phyB with PIF4. A Yeastthree hybrid assays showing that BBX11 enhances the interaction of phyB with BBX11 in yeast cells. B LCI assays showing that BBX11 enhances the interaction between phyB and PIF4 in living plant cells. Bar $=1 \mathrm{~cm}$. Data are the means SD of three independent biological replecates. Asterisks represent statistically significant differences $\left({ }^{* *} P<0.01\right.$; $\left.^{* *} P<0.001\right)$, as determined by Student's t-test

accumulated comparable PIF4 protein levels (Supplemental Fig. 4A). Consistent with previous findings (Yu et al. 2019), PIF4 was able to activate the proIAA19:LUC reporter in a transient transcriptional activation assay. Although BBX11 could not activate proIAA19:LUC, the activation on this reporter was significantly decreased when co-expressed PIF4 and BBX11 (Fig. 5A, B), suggesting that BBX11 inhibits the PIF4 transcriptional activation activity. We next performed chromatin immunoprecipitaiton (ChIP) experiments to assess whether BBX11 affects the PIF4 binding to target sites in plants. PIF4 associated with the IAA19 and IAA29 promoter regions, which was consistent with a previous study (Sun et al. 2013). The relative enrichment of PIF4 on IAA19 and IAA29 promoter regions was markedly increased in the proPIF4:PIF4-HA bbx11-1 transgenic seedling compared to that in the proPIF4:PIF4-HA seedlings (Fig. 5C). Together, these findings suggest that BBX11 negatively affects the binding of PIF4 to its target DNA cis-element. We next examined the PIF4-HA protein levels in proPIF4:PIF4-HA and proPIF4:PIF4-HA bbx11-1 seedlings grown in constant red light. proPIF4:PIF4-HA bbx11-1 seedlings accumulated more PIF4-HA protein compared with proPIF4:PIF4-HA (Supplemental Fig. 4B), further supporting the notion that BBX11 promotes the degradation of PIF4 in the prolonged red light (Fig. 3B). These results suggest that the accumulated PIF4 in red light-grown proPIF4:PIF4-HA $b b \times 11-1$ seedlings may also contribute to the increased binding of IAA19 and IAA29 promoter regions.

\section{BBX11 negatively regulates PIF4-controlled gene expression}

Considering that BBX11 negatively affected PIF4 abundance as well as its DNA binding affinity (Figs. 3B; 5AC), we thus examined the transcript levels of three PIF4controlled genes in $b b \times 11$ mutant and transgenic plants overexpressing $B B X 11$ grown in the constant red light. The expression of IAA19, IAA29, and YUC8 were significantly up-regulated in $b b \times 11-1$ and $b b \times 11-2$, but clearly down-regulated in YFP-BBX11 \#8, pif4-2 and bbx11-1 pif4-2 (Fig. 5D-F), implying that BBX11 negatively mediates the expression of PIF4 target genes in a PIF4dependent manner in Arabidopsis seedling grown in the red light.

To identify the potential target genes regulated by BBX11 or co-regulated by BBX11 and PIF4 at the whole genome level, we performed RNA sequencing (RNA-seq) using 4-d old red light-grown Col-0, bbx11-1 and pif4-2 seedlings. The expression of approximately 437 and 590 genes were significantly changed in bbx11-1 and pif4-2 compared to Col-0 respectively (Supplemental Table 1 and 2). Further analysis revealed that the transcription of 197 genes was significantly altered in both $b b \times 11-1$ and pif4-2 seedlings (Fig. 5G, H; Supplemental Table 3), indicating that BBX11 co-regulates a number of common target genes with PIF4 in red light-grown seedlings.

\section{$B B X 11$ genetically acts upstream of PIF4}

To examine the genetic links between $p h y B, B B X 11$ and PIF4, we analyzed the hypocotyl phenotypes of various 
A Effectors

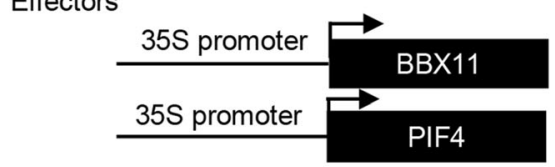

Reporter

\section{$\begin{array}{lll}\text { ProlAA19 Firefly luciferase } & \end{array}$}

Internal Control

35 S promoter

\section{$R n-L U C$}
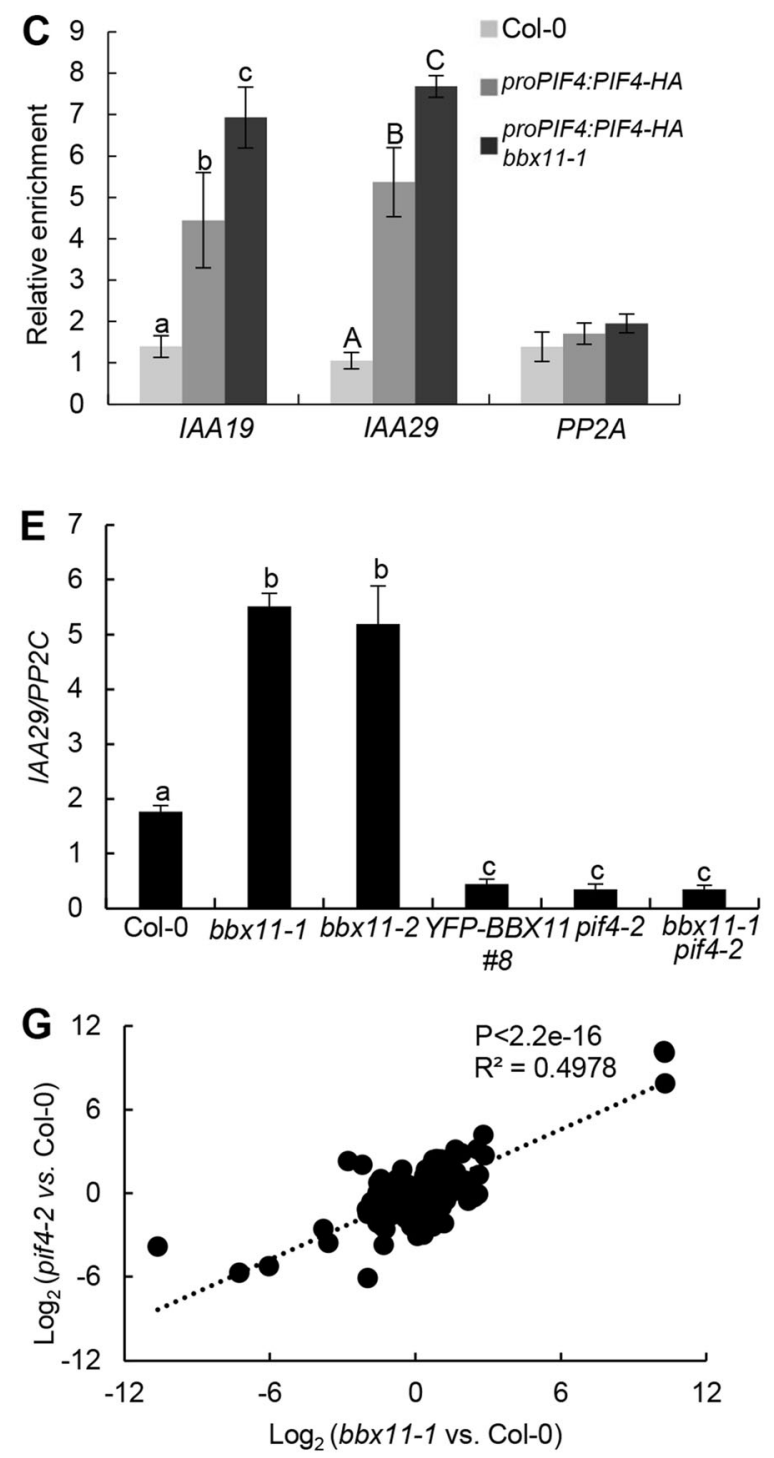
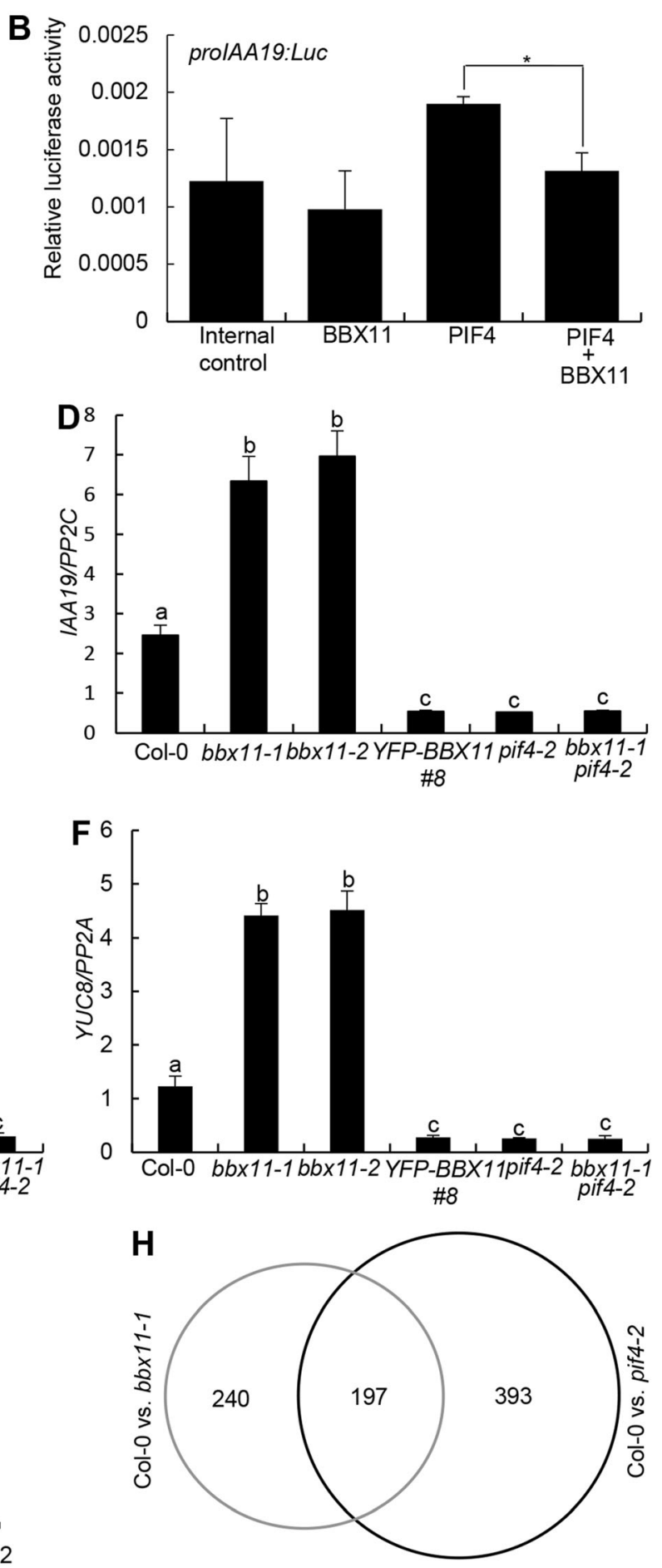
4Fig. 5 BBX11 represses the PIF4 binding to target sites. A Schematic representation of various constructs used in the transient transfection assay in Nicotiana benthamiana leaves. Arrow after the 35S promoter indicates the transcriptional start site. B Bar graphs showing that BBX11 represses the activation of the proIAA19:LUC by PIF4. Error bars represent SD of three independent transient transfections in Arabidopsis protoplasts. Asterisks represent statistically significant differences $\left({ }^{*} P<0.05\right)$, as determined by Student's $t$ test. C ChIP-qPCR assays showing that BBX11 is required for the PIF4 binding to the $I A A 19$ and IAA29 promoter regions in vivo. ChIP-qPCR assays were performed using 4-d-old Col-0, proPIF4:PIF4-HA, and proPIF4:PIF4-HA bbx11-1seedlings with anti-HA antibodies. Plants were grown in red $\left(94 \mu \mathrm{mol} / \mathrm{m}^{2} / \mathrm{s}\right)$ light for 4 days. The data represent means SD of three biological repeats. as determined by one-way ANOVA with Tukey's post hoc analysis. RT-qPCR analysis of IAA19 (D), IAA29 (E) and YUC8 (F) transcript levels in Col-0, bbx11-1, bbx11-2, YFP-BBX11 \#8, pif4-2 and bbx11-1 pif4-2 seedlings grown in red $\left(94 \mu \mathrm{mol} / \mathrm{m}^{2} / \mathrm{s}\right)$ for 4 days. Three biological replicates, each with three technical repeats, were performed. The data represent means SD of three biological repeats. Letters above the bars indicate significant differences $(P<0.05)$, as determined by one-way ANOVA with Tukey's post hoc analysis. G Scatterplot analysis of the fold change for genes showing differential expression either in $b b x 11-1$ or in pif4-2 compared to Col-0. $\mathbf{H}$ Venn diagram showing the number and overlap of genes whose expression was changed in bbx11-1 and pif4-2 seedlings

double mutants or transgenic seedlings grown in the red light. Consistent with results from previous studies (Neff and Chory 1998; Zhao et al. 2020), bbx11-1 and phyb-9 were longer than Col-0, whereas YFP-BBX11 \#8 was significantly shorter than Col-0 in the red light (Fig. 6A, B). The hypocotyl phenotypes of phyb-9 bbx111 resembled those of phyb-9 single mutant seedlings. YFP-BBX11 \#8 phyb-9 showed intermediate hypocotyls, which was longer than Col-0, bbx11-1, YFP-BBX11 \#8, but shorter than phyb-9 (Fig. 6A, B). These genetic data imply that BBX11 functions in phyB signaling. pif4-2 displayed shortened hypocotyl phenotypes (Fig. 6C, D), which was consistent with a previous study (Huq and Quail. 2002). The hypocotyl length of bbx11-1 pif4-2 was indistigshible from that of pif4-2 (Fig. 6C, D), suggesting that BBX11 genetically acts upstream of PIF4 with respect to the hypocotyl growth in the red light.

\section{DISCUSSION}

phyB perceives the red light signals and regulates red light-dependent cellular and developmental processes in Arabidopsis. Upon red light exposure, inactive phyB $\mathrm{pr}$ form is converted into active pfr form that associates with PIFs including PIF4, subsequently triggering their rapid phosphorylation, ubiquitination and degradation (Huq and Quail 2002; Lorrain et al. 2008; Leivar and
Monte 2014). As a b-HLH type transcription factor, PIF4 regulates a large number of genes' expression to promote cell elongation and hypocotyl growth (Franklin et al. 2011; Sun et al. 2012, 2013; Jin et al. 2020). Thus phyB-PIF4 module acts as a signaling hub by which plants sense and transduce red light signals to control seedling development. Here, we have identified BBX11 as an essential regulator of this signaling hub. BBX11 negatively regulates PIF4 abundance and its activity through directly interacting with phyB and PIF4 in prolonged red light conditions.

Numerous studies have documented that a variety of BBXs plays critical roles in the control of photomorphogenesis in plants (Xu 2020; Song et al. 2020a). Of these, BBX4 is a positive regulator of red light signaling. phyB associates with BBX4 and stabilizes its abundance in the red light, thus allowing accumulated BBX4 to form heterodimers with PIF3 to inhibit its biochemical activity towards downstream target genes (Heng et al. 2019a). Same as BBX4, BBX11 promotes red light-mediated photomorphogenesis (Zhao et al. 2020; Job and Datta 2020). Upon red light illumination, BBX11 interacted with both phyB and PIF4 (Figs. 1 and 2). This modulation appears to result in the repression of PIF4 action through at least two distinct molecular regulatory mechanisms. (i) BBX11 acts as an adaptor in the interaction between phyB and PIF4, and facilitates the association of phyB with PIF4. Hence BBX11 may promote the phyB-triggered PIF4 degradation following red light irradiation (Figs. 3 and 4). (ii) BBX11 forms heterodimers with PIF4 and impairs its DNA binding ability or competes with PIF4 for DNA binding (Fig. 5A-C). These findings demonstrate that BBX11 not only promotes the degradation of PIF4 but also inhibits its biochemical activity, thereby limiting PIF4 action in regulating target genes' expression and subsequent inhibition of hypocotyl growth. The hypocotyl length of phyb-9 bbx11-1 was similar to that of phyb-9, and YFPBBX11 \#8 phyb-9 displayed intermediate hypocotyl phenotypes in the red light. Moreover, the phenotypes of red light-grown $b b \times 11-1$ pif4-2 resembled those of pif4-2 single mutant seedlings (Fig. 6). These genetic data also support the conclusion that BBX11 acts in the phyBPIF4-mediated signaling. Both BBX4 and BBX11 function as positive regulators of phyB signaling, however, they work in concert with distinct PIFs and exert different modes of action in promoting this process. The exact genetic and molecular interconnection between BBX4 and BBX11 awaits additional investigation.

It is well established that phyB inactive pr conformer is converted into pfr form, which translocates from the cytosol to the nucleus and interacts with PIF4 to induce its degradation (Huq and Quail 2002; Lorrain et al. 

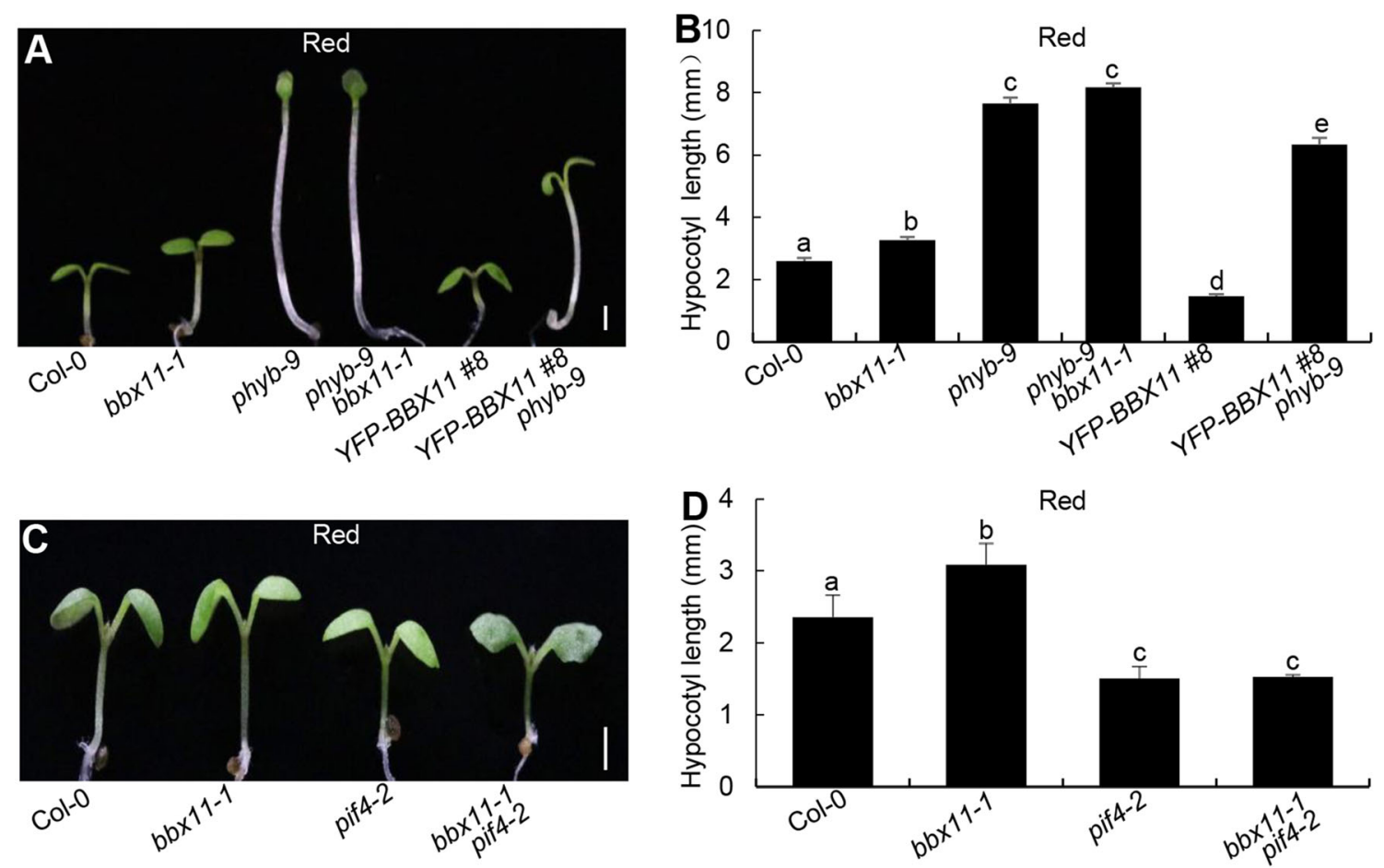

Fig. 6 BBX11 genetically acts upstream of PIF4. A, B Hypocotyl phenotype and length in 4-d-old Col-0, bbx1-1, phyb-9, YFP-BBX11 \#8 and YFP-BBX11 \#8 phyb-9 seedlings grown in red $\left(94 \mu \mathrm{mol} / \mathrm{m}^{2} / \mathrm{s}\right)$ light conditions. C, D Hypocotyl phenotype and length in 4-day-old Col-0,

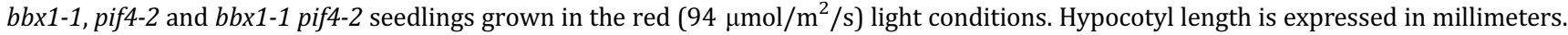
The data represent mean SE $(n \geq 60)$ of three biological replicates. Scale bars $=1 \mathrm{~mm}$. Letters above the bars indicate significant differences $(P<0.05)$, as determined by one-way ANOVA with Tukey's post hoc analysis

2008; Leivar and Monte 2014). These events may primarily occur at the early stage during the transition from dark to light, as phyB-imposed rapid PIF4 degradation implements within 10-60 min red light exposure (Lorrain et al. 2008; Zhang et al. 2017a; Yan et al. 2020). PIF4 re-accumulates to high abundant after prolonged $(>24 \mathrm{~h})$ red light irradiation (Zhang et al. 2017a; Yan et al. 2020), suggesting that PIF4 is required and indispensable for promoting hypocotyl growth under prolonged red light conditions. The transcription factor MYB30, which is a negative regulator of photomorphogenesis, promotes the PIF4 re-accumulation under prolonged red light irradiation (Yan et al. 2020). By contrast, BBX11 promotes PIF4 degradation under the same conditions (Fig. 4). These facts suggest that MYB30 and BBX11 have opposite effects on the PIF4 reaccumulation following prolonged red light exposure. It is therefore of interest in future studies to explore the exact links between MYB30 and BBX11 in controlling PIF4 protein level.

BBX11 exclusively interacted with PIF4, but not with PIF1, PIF3 and PIF5 (Fig. 2), implying it has a specialized role on PIF4 in phyB signaling. PIF4 plays pleiotropic roles in various cellular, physiological and developmental processes such as miRNA biogenesis, stomatal development, photomorphogenesis, thermomorphogenesis, phototropic response, brassinosteroid signaling, auxin biogenesis and signaling (Franklin et al. 2011; Oh et al. 2012; Lau et al. 2018; Sun et al. 2012, 2013, 2018). Thus BBX11 may be also involved in these diverse developmental and signaling pathways via modulating PIF4 action. In addition to phys, CRYPTOCHROME 1 (CRY1), COP1, DE-ETIOLATED 1 (DET1), CIRCADIAN CLOCK-ASSOCIATED 1 (CCA1), ENHANCED PHOTOMORPHOGENIC 2(EPP2), LATE ELONGATED HYPOCOTYL (LHY), TIMING OF CAB 1(TOC1), ELF3ELF4-LUX evening complex, COP1 SUPPRESSOR 4 (CSU4), HY5, COLD- REGULATED GENE 27 (COR27) and PIF4 itself regulate the PIF4 abundance, its transcriptional activity or transcription under specific conditions or at specific time points (Más et al. 2003; Nusinow et al. 2011; Nieto et al. 2015; Ma et al. 2016; Zhu et al. 2016, 2020; Huai et al. 2018; Zhao et al. 2018; Dong et al. 2020; Li et al. 2020; Zhai et al. 2020). Our study reveals that BBX11 is a novel negative regulator of PIF4. Most importantly, BBX11 plays dual role in inhibiting PIF4 function through promoting phyB-imposed PIF4 degradation as well as repressing the binding of PIF4 to its target sites. Altogether, these facts suggest a complex scenario whereby multiple factors converge on PIF4 in 
maintaining its appropriate abundance and/or transcriptional activation activity that ensures normal plant growth and development under natural conditions.

In summary, our results reveal that BBX11 is an essential regulator of the phyB-PIF4 signaling hub. BBX11 associates with both phyB and PIF4 that serves to enhance the interaction between phyB and PIF4, which in turn promoting the degradation of PIF4. On the other hand, BBX11 inhibits the DNA binding ability of PIF4. Consequently, these molecular events result in the repression of PIF4 function towards target genes (Fig. 7). Thus BBX11, acting downstream of phyB, negatively controls the PIF4 abundance as well as its biochemical activity in promoting photomorphogenesis in the red light. These observations demonstrate that the phyB-BBX11-PIF4 regulatory module acts as a central signaling hub that orchestrates red light-mediated seedling development.

\section{MATERIALS AND METHODS}

\section{Plant materials and growth conditions}

The phyb-9 (Neff and Chory 1998), pif4-2 (Leivar et al. 2008), bbx11-1, bbx11-2 mutants (Zhao et al. 2020), YFP-BBX11 \#8 (Zhao et al. 2020), and proPIF4:PIF4-HA (Zhang et al. 2017a) transgenic lines are of Col-0 ecotype. Double mutants/transgenic plants were generated by genetic crossing, and homozygous lines were verified by PCR genotyping or antibiotic screen. Seeds were surface sterilized with 30\% commercial Clorox bleach and sown on $1 \times$ Murashige and Skoog (MS) medium containing $1 \%$ sucrose and $0.8 \%$ agar. The seeds were stratified in darkness for 3 days at $4{ }^{\circ} \mathrm{C}$, then transferred to light chambers maintained at $22{ }^{\circ} \mathrm{C}$. The fluence rates of the light growth chambers were $100 \mu \mathrm{mol} / \mathrm{m}^{2} / \mathrm{s}$ for white light and $94 \mu \mathrm{mol} / \mathrm{m}^{2} / \mathrm{s}$ for a red light.

\section{Plasmid construction}

To generate $p B 42 A D-B B X 11$, the full-length of $B B X 11$ were amplified by PCR with the respective pairs of primers and then cloned into the EcoRI/XhoI sites of the $p B 42 A D$ vector (BD Clontech). To generate $p L e x A-p h y B$, pLexA-phyB-N, pLexA-phyB-C, pLexA-phyB-C1, pLexAphyB-C2, pLexA-BBX11, pLexA-BBX11(1-218), pLexABBX11 (1-97), pLeXA-BBX11 (84-286), pLexA-BBX11 (280-332) and pLexA-BBX11 4 (97-213), full-length $p h y B$, phyB-N(1-652), phyB-C(652-1172), phyB-C1(910-1172), phyB-C2(652-910), full-length $B B X 11, B B X 11(1-218)$, $B B X 11(1-97), \quad B B X 11(85-286), B B X 11(280-332)$ and $B B X 11$ lacking (98-212) fragments were amplified by PCR with a specific pair of primers and then cloned into the EcoRI/XhoI sites of the $p L e x A$ vector (BD Clontech). pB42AD-PIF1, $p$ B42AD-PIF3, $p B 42 A D$-PIF4, $p B 42 A D$-PIF5 (Dong et al. 2014), pGreenII0800-proIAA19:LUC (Yu et al. 2019), pEarley Gate-35S:myc-BBX11 (Zhao et al. 2020) constructs were described previously. The primers used

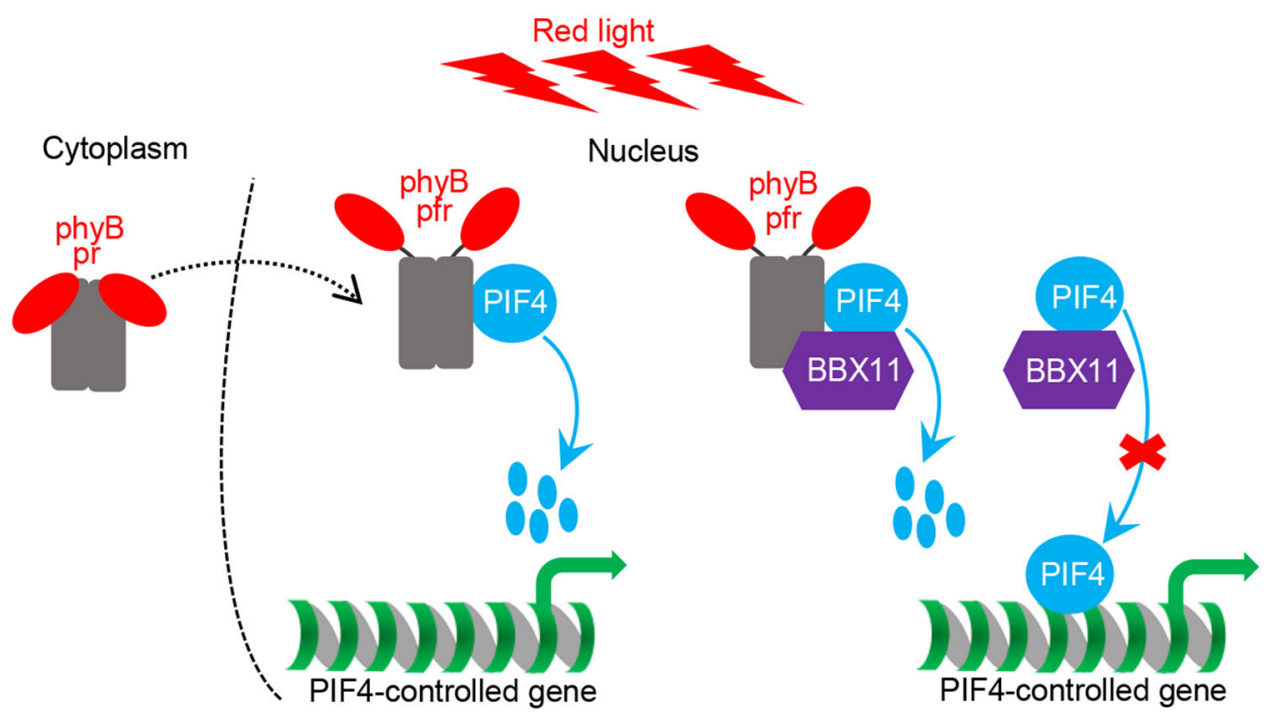

Fig. 7 A working model depicting that how BBX11 represses PIF4 function in the red light. Upon red light irradiation, pr form of phyB converts into pfr form that interacts with PIF4 to trigger its rapid degradation via the 26S proteasome system. On one hand, BBX11 enhances the interaction of phyB with PIF4 to promote the degradation of PIF4. On the other hand, BBX11 inhibits the binding of PIF4 to target DNA cis-elements. Hence, BBX11 represses the PIF4 function and PIF4-controlled gene expression to promote photomorphogenesis 
for plasmids construction were listed in Supplemental Table 4.

\section{Measurement of hypocotyl length}

To measure the hypocotyl length of seedlings, seeds were surface sterilized and sown on MS plates. The seeds were stratified at $4{ }^{\circ} \mathrm{C}$ in darkness for 3 days, and then placed in continuous white $\left(100 \mu \mathrm{mol} / \mathrm{m}^{2} / \mathrm{s}\right)$ light for $8 \mathrm{~h}$ to induce uniform germination. Then the seeds were transferred to continuous red $\left(94 \mu \mathrm{mol} / \mathrm{m}^{2} / \mathrm{s}\right)$ light conditions and incubated at $22{ }^{\circ} \mathrm{C}$ for 4 days. The hypocotyl length of seedlings was measured using ImageJ software.

\section{Co-Immunoprecipitation (Co-IP) assay}

Co-IP assays were performed as described previously (Lin et al. 2018). Agrobacterium strain GV3101 cells carrying the 35S:myc-BBX11, 35S:phyB-Flag or 35S:PIF4-Flag constructs were transiently infiltrated into Nicotiana benthamiana leaves. The plants were grown under long-day conditions (16 h Light/8 h Dark) for $3 \mathrm{~d}$ and lysed. Extracts were incubated with $4 \mu \mathrm{L}$ of anti-myc antibodies (1:250 v/v) (Sigma-Aldrich) coupled with $25 \mu \mathrm{L}$ of Protein-A Sepharose (GE Healthcare) for $3 \mathrm{~h}$ at $4{ }^{\circ} \mathrm{C}$. The Sepharose was washed three times using protein extraction buffer. The precipitates were eluted into $100 \mathrm{mM}$ glycine (pH 2.5) and $100 \mathrm{mM} \mathrm{NaCl}$, immediately neutralized by $2 \mathrm{M}$ Tris- $\mathrm{HCl}$ (pH 9.0), and $100 \mathrm{mM} \mathrm{NaCl}$, and then concentrated using StrataClean Resin (Stratagene) prior to immunoblot analysis.

\section{Immunoblot analysis}

For immunoblot analysis, Arabidopsis seedlings were homogenized in protein extraction buffer containing $100 \mathrm{mM} \mathrm{NaH}{ }_{2} \mathrm{PO}_{4}, 10 \mathrm{mM}$ Tris- $\mathrm{HCl}$ (pH8.0), $200 \mathrm{mM}$ $\mathrm{NaCl}, 8 \mathrm{M}$ Urea, $1 \mathrm{mM}$ PMSF, and $1 \times$ complete protease inhibitor cocktail (Roche). Primary antibodies used in this study were anti-PIF4 (Abicode Cat.R25344), anti-HA (MBL, Cat.M180-3), anti-myc (Sigma-Aldrich, Cat.M4439), and anti-Actin (Sigma-Aldrich, Cat.A0480).

\section{Yeast two-hybrid assays}

Yeast two-hybrid assays were performed using the Matchmaker LexA Two-Hybrid System as described in the Yeast Protocols Handbook (BD Clontech). The respective combinations of $p L e x A$ and $p B 42 A D$ fusion plasmids were co-transformed into yeast strain $E G Y 48$ containing $p 8 o p-L a c Z$ plasmid. The empty $p L e x A$ and $p B 42 A D$ vectors were co-transformed in parallel as negative controls. Transformants were selected and grown on SD/-His-Trp-Ura dropout plates at $30^{\circ} \mathrm{C}$. The transformants were grown on SD/-His-Trp-Ura dropout plates containing $80 \mathrm{mg} \mathrm{L}^{-1} \mathrm{X}$-gal for blue color development.

\section{Chromatin immunoprecipitation (ChIP)}

The ChIP assays were performed as described by $\mathrm{Xu}$ et al. (2016). Chromatin isolation was performed using Col-0, proPIF4:PIF4-HA and proPIF4:PIF4-HA bbx11-1 transgenic seedlings grown under constant red (94 $\mu \mathrm{mol} / \mathrm{m}^{2} / \mathrm{s}$ ) light for $4 \mathrm{~d}$. The resuspended chromatin was sonicated at $4{ }^{\circ} \mathrm{C}$ to 250 - to 500 -bp fragments. The sheared chromatin was immunoprecipitated, washed, reverse cross-linked, and finally amplified. About $10 \%$ of sonicated but nonimmunoprecipitated chromatin was reverse cross-linked and used as an input DNA control. Both immunoprecipitated DNA and input DNA were analyzed by real-time qPCR (Applied Biosystems). Monoclonal anti-HA antibody (MBL, Cat.M180-3), was used for the assays. All primers used for this assay are listed in Supplemental Table 4.

\section{Firefly LCI assay}

The firefly LCI transient expression assay was performed as described previously (Chen et al. 2008). Agrobacterium strain GV3101 strains carrying the binary plasmids of $p C A M B I A 1300-p h y B-n L U$ $C$ and $P C A M B I A 1300-C L U C-B B X 11$ or pCAMBIA1300PIF4-nLUC and pCAMBIA1300-cLUC-BBX11 were co-infiltrated into Nicotiana benthamiana leaves. The corresponding empty vector $P C A M B I A 1300$ nLUC or $P C A M B I A 1300-c L U C$ was infiltrated as a negative control. After a $2 \mathrm{~d}$ incubation in darkness at $22{ }^{\circ} \mathrm{C}$ and an additional $1 \mathrm{~d}$ incubation under a 16-h light/8-h dark photoperiod, the leaves were harvested, and $1 \mathrm{mM}$ D-luciferin (BD Monolight; BD Biosciences) solution was sprayed onto the tobacco leaves. Luciferase activity was measured with the LB 985 NightSHADE Spectrum imaging system (Berthold).

\section{Transient luciferase expression assays}

Nicotiana benthamiana plants grown in LD conditions (16 $\mathrm{h}$ light/8 $\mathrm{h}$ dark) were used for transient transactivation assay. Agrobacterium strain GV3101 cells carrying the 35S:myc-BBX11, 35S:PIF4-Flag or proIAA19:LUC constructs were transiently infiltrated into Nicotiana benthamiana leaves as indicated combinations. Firefly luciferase (LUC) and Renillia luciferase (Ren) were assayed using the Dual-Luciferase Reporter Assay System 
(Promega). The Ren gene driven by the cauliflower mosaic virus $35 \mathrm{~S}$ promoter was used as the control. The relative activity was expressed as a ratio of LUC/Ren.

\section{RNA-sequencing (RNA-seq) analysis}

Total RNA was extracted from the 4-d-old Col-0, bbx11-1 and pif4-2 seedlings grown in constant red light (94 $\mu \mathrm{mol} / \mathrm{m}^{2} / \mathrm{s}$ ) conditions. Then, mRNA sequencing libraries were constructed, and sequencing was performed using the Illumina HiSeq 2500 platform according to the manufacturer's instruction (HiSequation 2500 user guide) by Shanghai Hanyu-Bio. Three independent biological replicates were performed. RNASeq reads were mapped to Arabidopsis TAIR 10 using Hisat2 (version 2.05; Kim et al. 2015) software using default parameters. Then, raw reads for each gene were calculated by HTseq (Anders et al. 2015) before calculating differential gene expression. Then, differentially expressed genes among two conditions were identified using the general liner models method in the edgeR package (version 3.12.0; Robinson et al. 2010) with a false positive rate of 0.05 and a fold-change of 2 .

\section{Total RNA isolation and quantitative qRT-PCR}

4-d-old Arabidopsis seedlings grown under red light $\left(94 \mu \mathrm{mol} / \mathrm{m}^{2} / \mathrm{s}\right)$ conditions were used to isolate total RNA with an RNeasy Plant Mini Kit (Qiagen). cDNA synthesis reactions were performed with a $5 \times$ All-InOne RT MasterMix (Applied Biological Materials) according to the manufacturer's instructions. cDNA templates and primer sets were mixed with Hieff qPCR SYBR Green Master Mix (Yeasen), and real-time qPCR was performed on a StepOnePlus Real-time qPCR detection system (Applied Biosystems). Each experiment was performed at least three times with similar results and three technical replicates were performed for each sample. The expression levels were normalized to that of housekeeping gene PP2A. The primers used in RT-qPCR are listed in Supplemental Table 4.

\section{Statistical analysis}

Statistical analysis was performed using Microsoft Excel, GraphPad Prism version 5.0 or through an online website (http://astatsa.com/OneWay_Anova_with_ TukeyHSD/).

\section{Accession numbers}

Sequence data from this article can be found in the Arabidopsis Genome Initiative database or the GenBank/
EMBL libraries under the following accession numbers: phyB (AT2G18790); PIF4 (AT4G25350); BBX11 (AT2G47890). RNA-seq data have been deposited into the Gene Expression Omnibus with the following accession number: GSE166109.

Acknowledgements We thank Dr. Mingqiu Dai (Huazhong Agricultural University) for providing pGreenII0800-proIAA19:LUC, Dr Ove Nilsson (Swedish University of Agricultural Sciences) for providing proPIF4:PIF4-HA transgenic seeds and Dr. Xuncheng Wang for assistance in performing bioinformatics analysis. This work was supported by a grant from the National Natural Science Foundation of China (31970258), by start-up funding from Nanjing Agricultural University (to D.X.), by grants from Jiangsu "Innovative and Entrepreneurial Talent" program (to D.X.), Nanjing Science and Technology Innovation Program for Overseas Students (to D.X.), and the Jiangsu Collaborative Innovation Center for Modern Crop Production.

Authors' contributions ZS, YH, YB, YX, JL, XZ and YJ conducted the experiments. XWD and DX designed the experiments, analyzed the data, and wrote the article.

\section{Compliance with ethical standards}

Conflict of interest On behalf of all authors, the corresponding author states that there is no conflict of interest.

Open Access This article is licensed under a Creative Commons Attribution 4.0 International License, which permits use, sharing, adaptation, distribution and reproduction in any medium or format, as long as you give appropriate credit to the original author(s) and the source, provide a link to the Creative Commons licence, and indicate if changes were made. The images or other third party material in this article are included in the article's Creative Commons licence, unless indicated otherwise in a credit line to the material. If material is not included in the article's Creative Commons licence and your intended use is not permitted by statutory regulation or exceeds the permitted use, you will need to obtain permission directly from the copyright holder. To view a copy of this licence, visit http://creativecommons.org/ licenses/by/4.0/.

\section{References}

Anders S, Pyl PT, Huber W (2015) HTSeq-a Python framework to work with high-throughput sequencing data. Bioinformatics 31:166-169

Bursch K, Toledo-Ortiz G, Pireyre M, Lohr M, Braatz C, Johansson H (2020) Identification of BBX proteins as rate-limiting cofactors of HY5. Nat Plants 6:921-928

Chen H, Zou Y, Shang Y, Lin H, Wang Y, Cai R, Tang X, Zhou JM (2008) Firefly luciferase complementation imaging assay for protein-protein interactions in plants. Plant Physiol 146:368-376

Datta S, Hettiarachchi GH, Deng XW, Holm M (2006) Arabidopsis CONSTANS-LIKE3 is a positive regulator of red light signaling and root growth. Plant Cell 18:70-84

Datta S, Hettiarachchi C, Johansson H, Holm M (2007) SALT TOLERANCE HOMOLOG2, a B-box protein in Arabidopsis that 
activates transcription and positively regulates light-mediated development. Plant Cell 19:3242-3255

Datta S, Johansson H, Hettiarachchi C, Irigoyen ML, Desai M, Rubio V, Holm M (2008) LZF1/SALT TOLERANCE HOMOLOG3, an Arabidopsis B-box protein involved in light-dependent development and gene expression, undergoes COP1-mediated ubiquitination. Plant Cell 20:2324-2338

Dong J, Tang D, Gao Z, Yu R, Li K, He H, Terzaghi W, Deng XW, Chen H (2014) Arabidopsis DE-ETIOLATED 1 represses photomorphogenesis by positively regulating phytochrome-interacting factors in the dark. Plant Cell 26:3630-3645

Dong X, Yan Y, Jiang B, Shi Y, Jia Y, Cheng J, Shi Y, Kang J, Li H, Zhang D, Qi L, Han R, Zhang S, Zhou Y, Wang X, Terzaghi W, Gu H, Kang D, Yang S, Li J (2020) The cold response regulator CBF1 promotes Arabidopsis hypocotyl growth at ambient temperatures. EMBO J 39:e103630

Fan XY, Sun Y, Cao DM, Bai MY, Luo XM, Yang HJ, Wei CQ, Zhu SW, Sun Y, Chong K, Wang ZY (2012) BZS1, a B-box protein, promotes photomorphogenesis downstream of both brassinosteroid and light signaling pathways. Mol Plant 5:591-600

Franklin KA, Lee SH, Patel D, Kumar SV, Spartz AK, Gu C, Ye S, Yu P, Breen G, Cohen JD, Wigge PA, Gray WM (2011) Phytochromeinteracting factor 4 (PIF4) regulates auxin biosynthesis at high temperature. Proc Natl Acad Sci USA 108:20231-20235

Gangappa SN, Botto JF (2014) The BBX family of plant transcription factors. Trends Plant Sci 19:460-470

Gangappa SN, Crocco CD, Johansson H, Datta S, Hettiarachchi C, Holm M, Botto JF (2013) The Arabidopsis B-BOX protein BBX25 interacts with HY5, negatively regulating BBX22 expression to suppress seedling photomorphogenesis. Plant Cell 25:1243-1257

Galvao VC, Fankhauser C (2015) Sensing the light environment in plants: photoreceptors and early signaling steps. Curr Opin Neurobiol 34:46-53

Han X, Huang X, Deng XW (2020) The photomorphogenic central repressor COP1: conservation and functional diversification during evolution. Plant Comm 1:100044

Heng Y, Jiang Y, Zhao X, Zhou H, Wang X, Deng XW, Xu D (2019a) BBX4, a phyB interacting and -modulated regulator, directly interacts with PIF3 to fine tune red light-mediated photomorphogenesis. Proc Natl Acad Sci USA 116:26049-26056

Heng Y, Lin F, Jiang Y, Ding M, Yan T, Lan H, Zhou H, Zhao X, Xu D, Deng XW (2019b) B-box containing proteins BBX30 and BBX31, acting downstream of HY5, negatively regulate photomorphogenesis in Arabidopsis. Plant Physiol 180:497-508

Holtan HE, Bandong S, Marion CM, Adam L, Tiwari S, Shen Y, Maloof JN, Maszle DR, Ohto MA, Preuss S, Meister R, Petracek M, Repetti PP, Reuber TL, Ratcliffe OJ, Khanna R (2011) BBX32, an Arabidopsis B-Box protein, functions in light signaling by suppressing HY5-regulated gene expression and interacting with STH2/BBX21. Plant Physiol 156:2109-2123

Huai J, Zhang X, Li J, Ma T, Zha P, Jing Y, Lin R (2018) SEUSS and PIF4 coordinately regulate light and temperature signaling pathways to control plant growth. Mol Plant 11:928-942

Huq E, Quail PH (2002) PIF4, a phytochrome-interacting bHLH factor, functions as a negative regulator of phytochrome $\mathrm{B}$ signaling in Arabidopsis. EMBO J 21:2441-2450

Jiao Y, Lau OS, Deng XW (2007) Light-regulated transcriptional networks in higher plants. Nat Rev Genet 8:217-230

Jin H, Lin J, Zhu Z (2020) PIF4 and HOOKLESS1 impinge on common transcriptome and isoform regulation in thermomorphogenesis. Plant Comm 1:100034

Jing Y, Lin R (2020) Transcriptional regulatory network of the light signaling pathways. New Phytol 227:683-697
Job N, Yadukrishnan P, Bursch K, Datta S, Johansson H (2018) Two B-Box proteins regulate photomorphogenesis by oppositely modulating HY5 through their diverse C-terminal domains. Plant Physiol 176:2963-2976

Job N, Datta S (2020) PIF3-HY5 module regulates BBX11 to suppress protochlorophyllide levels in dark and promote photomorphogenesis in light. New Phytol. https://doi.org/10. 1111/nph.17149

Khanna R, Kronmiller B, Maszle DR, Coupland G, Holm M, Mizuno T, Wu SH (2009) The Arabidopsis B-box zinc finger family. Plant Cell 21:3416-3420

Kim D, Langmead B, Salzberg SL (2015) HISAT: A fast spliced aligner with low memory requirements. Nat Methods 12:357-360

Lau OS, Song Z, Zhou Z, Davies KA, Chang J, Yang X, Wang S, Lucyshyn D, Tay IHZ, Wigge PA, Bergmann DC (2018) Direct control of SPEECHLESS by PIF4 in the high-temperature response of stomatal development. Curr Biol 28:1273-1280

Leivar P, Monte E, Sady BA, Carle C, Storer A, Alonso JM, Ecker JR, Quail PH (2008) The Arabidopsis phytochrome-interacting factor PIF7, together with PIF3 and PIF4, regulates responses to prolonged red light by modulating phyB levels. Plant Cell 20:337-352

Leivar P, Monte E (2014) PIFs: systems integrators in plant development. Plant Cell 26:56-78

Li J, Li G, Wang H, Deng XW (2011) Phytochrome signaling mechanisms. Arabidopsis 9:e0148

Li X, Liu C, Zhao Z, Ma D, Zhang J, Yang Y, Liu Y, Liu H (2020) COR27 and COR28 are novel regulators of the COP1-HY5 regulatory hub and photomorphogenesis in arabidopsis. Plant Cell 32:3139-3154

Lin F, Jiang Y, Li J, Yan T, Fan L, Liang J, Chen ZJ, Xu D, Deng XW (2018) B-BOX DOMAIN PROTEIN28 negatively regulates photomorphogenesis by repressing the activity of transcription factor HY5 and undergoes COP1-mediated degradation. Plant Cell 30:2006-2019

Lorrain S, Allen T, Duek PD, Whitelam GC, Fankhauser C (2008) Phytochrome-mediated inhibition of shade avoidance involves degradation of growth-promoting bHLH transcription factors. Plant J 53:312-323

Ma D, Li X, Guo Y, Chu J, Fang S, Yan C, Noel JP, Liu H (2016) Cryptochrome 1 interacts with PIF4 to regulate high temperature-mediated hypocotyl elongation in response to blue light. Proc Natl Sci Acad USA 113:224-229

Más P, Alabadí D, YanovskyMJ OT, Kay SA (2003) Dual role of TOC1 in the control of circadian and photomorphogenic responses in Arabidopsis. Plant Cell 15:223-236

Nieto C, López-Salmerón V, Davière JM, Prat S (2015) ELF3-PIF4 interaction regulates plant growth independently of the evening complex. Curr Biol 25:187-193

Nusinow DA, Helfer A, Hamilton EE, King JJ, Imaizumi T, Schultz TF, Farré EM, Kay SA (2011) The ELF4-ELF3-LUX complex links the circadian clock to diurnal control of hypocotyl growth. Nature 475:398-402

Neff M, Chory J (1998) Genetic interactions between phytochrome A, phytochrome B, and cryptochrome 1 during Arabidopsis development. Plant Physiol 118:27-35

Oh E, Zhu JY, Wang ZY (2012) Interaction between BZR1 and PIF4 integrates brassinosteroid and environmental responses. Nat Cell Biol 14:802-809

Park E, Kim Y, Choi G (2018) Phytochrome B requires PIF degradation and sequestration to induce light responses across a wide range of light conditions. Plant Cell 30:1277-1292 
Paik I, Huq E (2019) Plant photoreceptors: Multi-functional sensory proteins and their signaling networks. Semin Cell Dev Biol 92:114-121

Pham VN, Kathare PK, Huq E (2018) Phytochromes and phytochrome interacting factors. Plant Physiol 176:1025-1038

Robinson MD, McCarthy DJ, Smyth GK (2010) edgeR: A Bioconductor package for differential expression analysis of digital gene expression data. Bioinformatics 26:139-140

Sun J, Qi L, Li Y, Chu J, Li C (2012) PIF4-mediated activation of YUCCA8 expression integrates temperature into the auxin pathway in regulating Arabidopsis hypocotyl growth. PLoS Genet 8:e1002594

Sun J, Qi L, Li Y, Zhai Q, Li C (2013) PIF4 and PIF5 transcription factors link blue light and auxin to regulate the phototropic response in Arabidopsis. Plant Cell 25:2102-2114

Sun Z, Li M, Zhou Y, Guo T, Liu Y, Zhang H, Fang Y (2018) Coordinated regulation of Arabidopsis microRNA biogenesis and red light signaling through Dicer-like 1 and phytochrome-interacting factor 4. PLoS Genet 14:e1007247

Song Z, Bian Y, Liu J, Sun Y, Xu D (2020a) B-box proteins: pivotal players in light-mediated development in plants. J Integr Plant Biol 62:1293-1309

Song Z, Yan T, Liu J, Bian Y, Heng Y, Lin F, Jiang Y, Deng XW, Xu D (2020b) BBX28/BBX29, HY5 and BBX30/31 form a feedback loop to fine-tune photomorphogenic development. Plant J 104:377-390

Sharrock RA, Quail PH (1989) Novel phytochrome sequences in Arabidopsis thaliana: structure, evolution, and differential expression of a plant regulatory photoreceptor family. Genes Dev 3:1745-1757

Vaishak KP, Yadukrishnan P, Bakshi S, Kushwaha AK, Ramachandran H, Job N, Babu D, Datta S (2019) The B-box bridge between light and hormones in plants. J Photochem Photobiol B 191:164-174

Wang CQ Sarmast MK, Jiang J, Dehesh K (2015) The transcriptional regulator BBX19 promotes hypocotyl growth by facilitating COP1-mediated EARLY FLOWERING3 degradation in Arabidbopsis. Plant Cell 27:1128-1139

Wei H, Kong D, Yang J, Wang H (2020) Light regulation of stomatal development and patterning: shifting the paradigm from Arabidopsis to grasses. Plant Comm 1:100030

Wu Q Kuang K, Lyu M, Zhao Y, Li Y, Li J, Pan Y, Shi H, Zhong S (2020) Allosteric deactivation of PIFs and EIN3 by microproteins in light control of plant development. Proc Natl Acad Sci USA 117:18858-18868

Xu D (2020) COP1- and BBXs-HY5-mediated light signal transduction in plants. New Phytol 228:1748-1753

Xu D, Jiang Y, Li J, Lin F, Holm M, Deng XW (2016) BBX21, an Arabidopsis B-box protein, directly activates HY5 and is targeted by COP1 for $26 \mathrm{~S}$ proteasome-mediated degradation. Proc Natl Acad Sci USA 113:7655-7660

Xu D, Jiang Y, Li J, Lin F, Holm M, Deng XW (2018) B-box domain protein BBX21 promotes photomorphogenesis. Plant Physiol 176:2365-2375

Yadav A, Bakshi S, Yadukrishnan P, Lingwan M, Dolde U, Wenkel S, Masakapalli SK, Datta S (2019) The B-Box-containing microprotein miP1a/BBX31 regulates photomorphogenesis and UV-B protection. Plant Physiol 179:1876-1892

Yadav A, Singh D, Lingwan M, Yadukrishnan P, Masakapalli SK, Datta S (2020) Light signaling and UV-B mediated plant growth regulation. J Integr Plant Biol 62:1270-1292

Yadukrishnan P, Rahul PV, Ravindran N, Bursch K, Johansson H, Datta S (2020) CONSTITUTIVELY PHOTOMORPHOGENIC1 promotes ABA-mediated inhibition of post-germination seedling establishment. Plant J 103:481-496

Yadukrishnan P, Datta S (2020) Light and abscisic acid interplay in early seedling development. New Phytol. https://doi.org/10. 1111/nph.16963

Yan Y, Li C, Dong X, Li H, Zhang D, Zhou Y, Jiang B, Peng J, Qin X, Cheng J, Wang X, Song P, Qi L, Zheng Y, Li B, Terzaghi W, Yang S, Guo Y, Li J (2020) MYB30 is a key negative regulator of Arabidopsis photomorphogenic development that promotes PIF4 and PIF5 protein accumulation in the light. Plant Cell 32:2196-2215

Yang L, Liu S, Lin R (2020) The role of light in regulating seed dormancy and germination. J Integr Plant Biol 62:1310-1326

Yu X, Dong J, Deng Z, Jiang Y, Wu C, Qin X, Terzaghi W, Chen H, Dai M, Deng XW (2019) Arabidopsis PP6 phosphatases dephosphorylate PIF proteins to repress photomorphogenesis. Proc Natl Acad Sci USA 116:20218-20225

Yu Y, Liu H (2020) Coordinated shoot and root responses to light signaling in Arabidopsis. Plant Comm 1:100026

Zhang B, Holmlund M, Lorrain S, Norberg M, Bakó L, Fankhauser C, Nilsson O (2017a) BLADE-ON-PETIOLE proteins act in an E3 ubiquitin ligase complex to regulate PHYTOCHROME INTERACTING FACTOR 4 abundance. eLife 6:e26759

Zhang X, Shang F, Huai J, Xu G, Tang W, Jing Y, Lin R (2017b) A PIF1/PIF3-HY5-BBX23 transcription factor cascade affects photomorphogenesis. Plant Physiol 174:2487-2500

Zhang Z, Ji R, Li H, Zhao T, Liu J, Lin C, Liu B (2014) CONSTANSLIKE 7 (COL7) is involved in phytochrome $B$ (phyB)mediated light-quality regulation of auxin homeostasis. Mol Plant 7:1429-1440

Zhai H, Xiong L, Li H, Lyu X, Yang G, Zhao T, Liu J, Liu B (2020) Cryptochrome 1 inhibits shoot branching by repressing the self-activated transciption loop of PIF4 in Arabidopsis. Plant Comm 1:100042

Zhao X, Jiang Y, Li J, Huq E, Chen ZJ, Xu D, Deng XW (2018) COP1 SUPPRESSOR 4 promotes seedling photomorphogenesis by repressing CCA1 and PIF4 expression in Arabidopsis. Proc Natl Acad Sci USA 115:11631-11636

Zhao X, Heng Y, Wang X, Deng XW, Xu D (2020) A positive feedback loop of BBX11-BBX21-HY5 promotes photomorphogenic development in Arabidopsis. Plant Comm 1:100045

Zhu W, Zhou H, Lin F, Zhao X, Jiang Y, Xu D, Deng XW (2020) COLDREGULATED GENE 27 integrates signals from light and circadian rhythm in promoting hypocotyl growth in Arabidopsis. Plant Cell 32:3155-3169

Zhu JY, Oh E, Wang T, Wang ZY (2016) TOC1-PIF4 interaction mediates the circadian gating of thermoresponsive growth in Arabidopsis. Nat Commun 7:13692 